\title{
Usefulness of preoperative magnetic resonance spectroscopy in improving the safety of a living liver donor
}

\author{
Jae Ryong Shim, Hyo Jung Ko, Tae Beom Lee, Byung Hyun Choi, Kwangho Yang, Je Ho Ryu
}

Department of Surgery, Pusan National University Yangsan Hospital, Yangsan, Korea

Background: The steatosis of graft liver is an important factor in liver transplantation that determines the graft function in the recipient and the recovery of the remnant liver in the living donor. We analyzed the data of living donor from our center to evaluate whether magnetic resonance imaging (MRI) and magnetic resonance spectroscopy (MRS) can replace liver biopsy.

Methods: From May 2010 to May 2019, total 239 living donor data was collected. Eighty-four patients who had no MRI or MRS data were excluded. The result of preoperative liver biopsy was compared to preoperative MRI and MRS data. The steatosis was defined by the degree of macrosteatosis.

Results: The MRI fat fraction was a good parameter in predicting fatty changes between normal and fatty liver groups $(3.09 \% \pm 3.38 \%, 7.48 \% \pm 4.07 \% ; \mathrm{P}<0.001)$. The MRS was also a good parameter for predicting fatty changes between normal and fatty liver groups $(2.09 \% \pm 1.43 \%, 6.89 \% \pm 2.68 \% ; P<0.001)$. Linear regression showed that pathological results were significantly correlated with MRS $\left(P<0.001, R^{2}=0.604\right)$, but not with MRI $\left(P<0.001, R^{2}=0.227\right)$.

Conclusions: MRS has several benefits for quantifying hepatic steatosis during a living donor liver transplantation evaluation, including no radiation exposure, and a noninvasive procedure. Moreover, preoperative MRS can determine an anatomical variation of the bile duct, which helps improve the safety of the living donor. However, more clinical data and further studies are needed to ensure that preoperative MRI is essential.

Corresponding author: Jae Ryong Shim

E-mail: zombiepr@naver.com

(c) The Korean Society for Transplantation

This is an Open Access article distributed under the terms of the Creative Commons Attribution Non-Commercial License (http://creativecommons.org/licenses/by-nc/4.0/) which permits unrestricted non-commercial use, distribution, and reproduction in any medium, provided the original work is properly cited. 